\title{
An improved growth medium for enhanced inoculum production of the plant growth-promoting fungus Serendipita indica
}

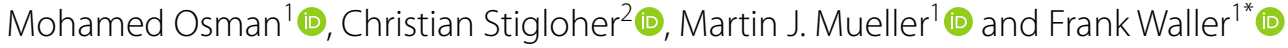

\begin{abstract}
Background: The plant endophytic fungus Serendipita indica colonizes roots of a wide range of plant species and can enhance growth and stress resistance of these plants. Due to its ease of axenic cultivation and its broad host plant range including the model plant Arabidopsis thaliana and numerous crop plants, it is widely used as a model fungus to study beneficial fungus-root interactions. In addition, it was suggested to be utilized for commercial applications, e.g. to enhance yield in barley and other species. To produce inoculum, S. indica is mostly cultivated in a complex HillKäfer medium (CM medium), however, growth in this medium is slow, and yield of chlamydospores, which are often used for plant root inoculation, is relatively low.
\end{abstract}

Results: We tested and optimized a simple vegetable juice-based medium for an enhanced yield of fungal inoculum. The described vegetable juice (VJ) medium is based on commercially available vegetable juice and is easy to prepare. VJ medium was superior to the currently used CM medium with respect to biomass production in liquid medium and hyphal growth on agar plates. Using solid VJ medium supplemented with sucrose (VJS), a high amount of chlamydospores developed already after 8 days of cultivation, producing significantly more spores than on CM medium. Use of $\mathrm{VJ}$ medium is not restricted to $\mathrm{S}$. indica, as it also supported growth of two pathogenic fungi often used in plant pathology experiments: the ascomycete Fusarium graminearum, the causal agent of Fusarium head blight disease on wheat and barley, and Verticillium longisporum, the causal agent of verticillium wilt.

Conclusions: The described VJ medium is recommended for streamlined and efficient production of inoculum for the plant endophytic fungus Serendipita indica and might prove superior for the propagation of other fungi for research purposes.

Keywords: Serendipita indica, Plant root endophyte, Inoculum production, Complex medium, Aspergillus medium, Vegetable juice, Plant growth promotion

\section{Background}

Serendipita indica, first described as Piriformospora indica [1], is a facultative root endophytic fungus belonging to the basidiomycotal order Sebacinales. Within this

\footnotetext{
*Correspondence: Frank.Waller@uni-wuerzburg.de

1 Julius-Von-Sachs Institute of Biosciences, Biocenter, Pharmaceutical

Biology, Julius-Maximilians-Universität Würzburg, Julius-von-Sachs-Platz 2, 97082 Würzburg, Germany

Full list of author information is available at the end of the article
}

clade, lifestyles range from saprotrophic to endophytic and obligate biotrophic root-colonizing fungi. Members of Sebacinales are often root-interacting endophytes involved in associations with a very wide range of plant species, and they are globally distributed [2, 3].

Serendipita indica colonizes roots of a vast number of different plant species. It is able to enhance the growth and yield of mono- and dicotyledonous plants, and enhances host plant resistance to biotic and abiotic stresses [2, 4-6]. Due to these positive effects on host

(c) The Author(s) 2020. This article is licensed under a Creative Commons Attribution 4.0 International License, which permits use, sharing, adaptation, distribution and reproduction in any medium or format, as long as you give appropriate credit to the original author(s) and the source, provide a link to the Creative Commons licence, and indicate if changes were made. The images or other third party material in this article are included in the article's Creative Commons licence, unless indicated otherwise in a credit line to the material. If material is not included in the article's Creative Commons licence and your intended use is not permitted by statutory regulation or exceeds the permitted use, you will need to obtain permission directly from the copyright holder. To view a copy of this licence, visit http://creativeco mmons.org/licenses/by/4.0/. The Creative Commons Public Domain Dedication waiver (http://creativecommons.org/publicdomain/ zero/1.0/) applies to the data made available in this article, unless otherwise stated in a credit line to the data. 
plants, and its ability to grow in axenic culture, it became a model fungus for studying the physiology and molecular basis of symbiotic plant-microbe interactions [7-10]. It was also suggested for diverse applications, e.g. as a biofertilizer and biocontrol agent $[6,11]$, for improvement of plant cell culture [12], or in vitro cultivated medical plants [13].

Serendipita indica can be propagated axenically on various complex and minimal substrates in the absence of host plants [4]. S. indica chlamydospores (asexual resting spores) germinate and form hyphae which resemble a string of beads (monilioid hyphae), which, after a few days, develop chlamydospores again.

A range of media have been proposed for axenic $S$. indica propagation, but these media are complex and can be somewhat laborsome to prepare with particular technical requirements $[14,15]$ or are easy to prepare and economical, but less productive [16]. The most commonly used medium is a Hill-Käfer complex medium (CM) containing 25 different macro- and micro-ingredients $[14,17]$. Using this CM agar medium, S. indica typically requires 3 weeks to cover a $9.5 \mathrm{~cm}$ Petri dish with hyphae after plug inoculation [18]. Different modifications of $\mathrm{CM}$ medium have been suggested for improving growth of $S$. indica axenically: reduction of the working volume of liquid medium, using different carbohydrate sources and adjustments of their concentrations; and optimizing agitation speed of liquid cultures [14, 15]. However, these efforts added more complexity to medium preparation or growth conditions and had only limited success in increasing fungal growth.

We therefore aimed at developing a growth medium for $S$. indica, which is simple to prepare while providing high inoculum yield. We rationalized that a medium based on plant tissue could be suitable for the facultative saprophyte $S$. indica and started using vegetable juice as a base for the medium. The commercially available V8 juice (Campbell Soup Co.) is the main component of different media used for propagation of plant fungal pathogens, e.g. for sporangia induction in Phytophthora [19]. In this study, we describe an optimized medium based on vegetable juice, which is simple to prepare and yields high amounts of inoculum from the root-colonizing model fungus S. indica.

\section{Results}

\section{Serendipita indica biomass production in liquid medium}

To optimize yield of the plant root colonizing fungus $S$. indica, we tested different media for fungal inoculum production. 10 days after inoculation of $100 \mathrm{ml}$ of medium with $S$. indica, the commonly used complex Kaefer-Hill medium (CM) yielded $1.6 \mathrm{~g}$ (fresh weight). In comparison, a vegetable juice (VJ) medium enhanced $S$. indica biomass production by a factor of around 13 (Fig. 1a). The basal VJ medium has a simple composition of $15 \%(\mathrm{v} / \mathrm{v})$ V8-juice (Campbell Soup Corp.; a commercially available vegetable juice) and $5 \mathrm{mM} \mathrm{CaCO}$. Omission of $\mathrm{CaCO}_{3}$, which is not only
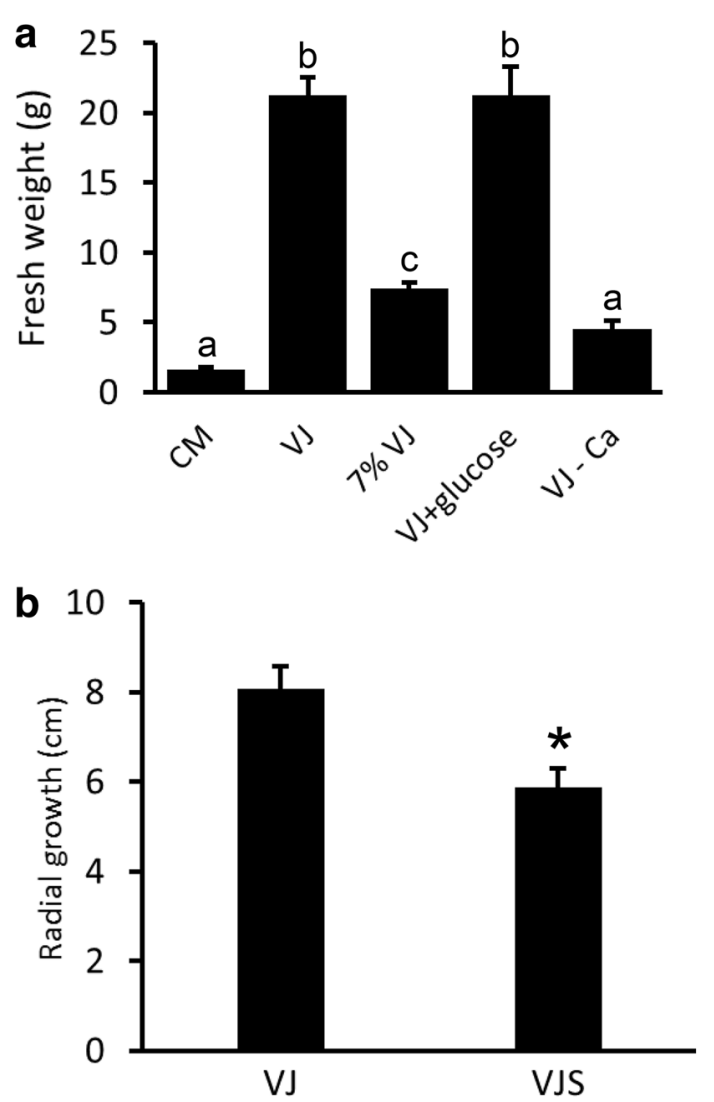

Fig. 1 Serendipita indica biomass production in different media. a The fungus was grown in $100 \mathrm{ml}$ of medium in $300 \mathrm{ml}$ Erlenmeyer flasks for 10 days at room temperature $\left(20 \pm 3^{\circ} \mathrm{C}\right)$ on a shaker (70 rpm). Mycelium was retrieved using Miracloth and fresh weight was determined subsequently. Media used: Complex medium 'CM'; Vegetable juice 'VJ' medium: $150 \mathrm{ml}$ V 8 juice, $850 \mathrm{ml}$ distilled water and $5 \mathrm{mM} \mathrm{CaCO}_{3}{ }^{\prime} 7 \% \mathrm{VJ}$ ' medium': VJ medium with $70 \mathrm{ml}$ instead of $150 \mathrm{ml}$ V8 juice;'VJ + D-glucose': VJ medium supplemented with $10 \mathrm{~g} / \mathrm{L}$ D-glucose;'VJ-Ca' medium: $\mathrm{VJ}$ medium without $\mathrm{CaCO}_{3}$. Values are means of three cultures, with error bars indicating standard deviation. The values were subjected to a one-way analysis of variance (ANOVA) followed by Tukey's post-hoc-test. Mean values significantly different at an alpha level of 0.05 are indicated by different letters. Experiments were independently reproduced at least three times with similar results. $\mathbf{b}$ Diameter of fungal colonies on agar plates prepared with VJ medium or VJS medium. VJS medium is VJ medium supplemented with $40 \mathrm{~g} / \mathrm{L}$ sucrose and with $\mathrm{CaCO}_{3}$ to a final concentration of $20 \mathrm{mM}$. Values are means of three cultures, with error bars indicating standard deviation. A Student's t-test was calculated, and the asterisk indicates a p-value lower 0.05 . Experiments were independently reproduced at least three times with similar results 
important as a medium component per se, but also to increase the $\mathrm{pH}$ to mitigate V8 acidity, led to a significant drop in fungal biomass (Fig. 1a). Similarly, decreasing V8 juice content in the medium from 15 to $7 \%(\mathrm{v} / \mathrm{v})$ reduced fungal biomass yield significantly (Fig. 1a). A vegetable juice content higher than $15 \%$ is not recommended because this leads to a higher viscosity and particle content, which would necessitate additional steps like centrifugation or filtration to retrieve fungal inoculum. Addition of glucose to the VJ medium did not result in a further increase of fungal biomass in liquid culture (Fig. 1a). We also tested a different commercial brand of vegetable juice, which gave comparable results to V8 juice with respect to fungal biomass production (data not shown).

\section{Growth of S. indica on agar medium for chlamydospore production}

To produce $S$. indica chlamydospores for plant root inoculation, $S$. indica is commonly cultivated on agar plates using CM medium. We compared growth of $S$. indica on $\mathrm{CM}$ agar with $\mathrm{VJ}$ medium. VJ agar plates were completely covered by fungal mycelium 8-10 days post inoculation, while growth on CM medium was significantly slower (Fig. 2a), requiring about three weeks to completely cover the plate with mycelium. Cultivation under continuous light or in darkness had only minor effects on fungal growth, both with $\mathrm{VJ}$ and $\mathrm{CM}$ medium (Additional file 1: Fig. 1). Addition of sucrose to the VJ medium did not increase, but rather decrease colony diameter (Fig. 1b). However, sucrose in the VJ medium was required for a high chlamydospore yield on this medium: At $8 \mathrm{dpi}$, a low number of spores was recovered from $S$. indica grown on $\mathrm{CM}$ at this early time point corresponding to the small colony diameter (Fig. 2a). Surprisingly, VJ basal medium yielded only few spores although plates were completely covered with fungal mycelium. Adding $4 \%$ sucrose and increasing $\mathrm{CaCO}_{3}$ concentration to $20 \mathrm{mM}$ in the $\mathrm{VJ}$ medium, however, led to a higher spore production compared to both the basal VJ and CM medium (Fig. 2b). We termed this medium VJS (for vegetable juice sucrose medium).

\section{Effect of Myo-inositol on S. indica growth on agar plates}

To test the hypothesis that the cause of $S$. indica growth enhancement is because of the Myo-inositol content of V8 $[20,21]$ a solid medium (yeast extract peptone agar) with $1 \%$ Myo-inositol or with $1 \%$ glucose was tested. However, the presence of Myo-inositol reduced colony diameter. Moreover, 1\% Myo-inositol attenuated
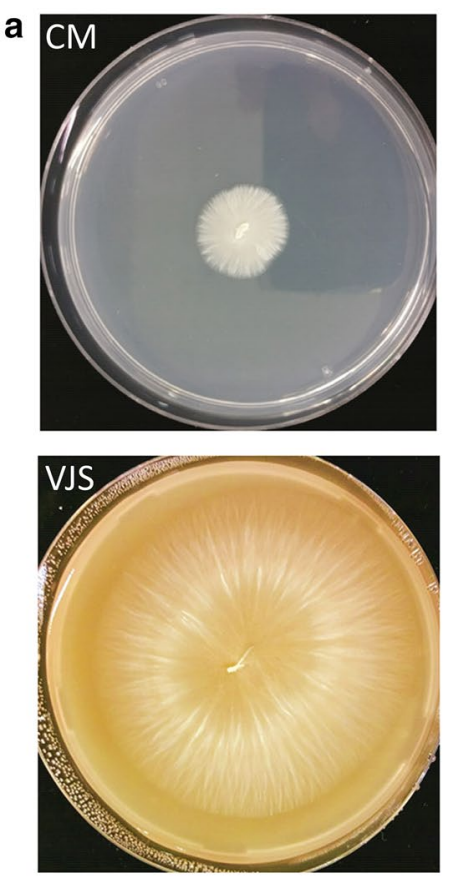

b

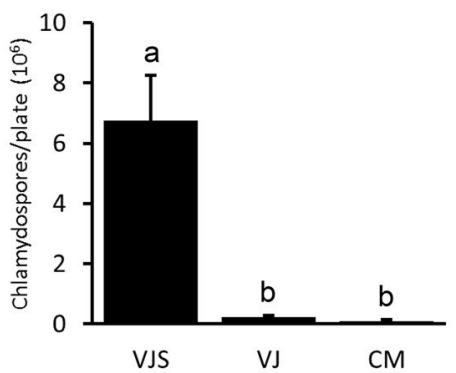

Fig. 2 S. indica chlamydospore production on agar medium. a Image of fungal growth on CM agar and VJS agar 8 days after inoculation with an agar plug. Standard Petri dishes with a diameter of $9 \mathrm{~cm}$ were used and kept at room temperature $\left(20 \pm 3^{\circ} \mathrm{C}\right)$ in the dark. $\mathbf{b}$ Amount of chlamydospores harvested from agar plates. Chlamydospores were obtained by washing the surface of the plates with $15 \mathrm{ml}$ of distilled water using a spatula, and subsequent filtering through Miracloth to remove hyphae and debris. After appropriate dilution, chlamydospores were counted using a microscope and a Fuchs-Rosenthal counting chamber. Values are means from three plates, with error bars indicating standard error. The values were subjected to a one-way analysis of variance (ANOVA) followed by Tukey's post-hoc-test. Mean values significantly different at an alpha level of 0.05 are indicated by different letters. Experiments were independently reproduced at least three times with similar results

aerial mycelia in contrast to the control, which was rich in superficial and aerial mycelia (Additional file 1: Fig. 2).

\section{VJ medium is highly suitable for inoculum production of Fusarium graminearum and Verticillium longisporum} We tested growth of the important wheat pathogen F. graminearum on $\mathrm{VJ}$ agar medium and recorded the 


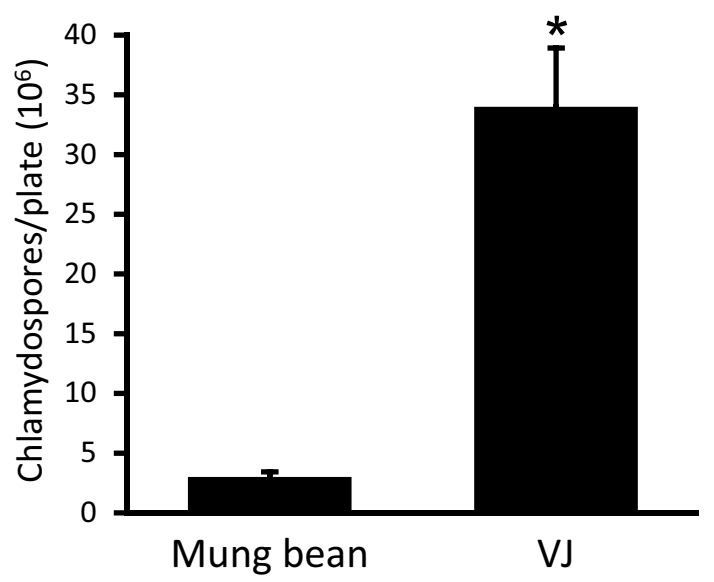

Fig. 3 Fusarium graminearum macroconidia production on VJ agar F. graminearum was grown on Mung bean agar medium or on VJ agar medium for 14 days in the dark. Total number of macroconidia recovered from a single plate is presented. Values are means from three plates, with error bars indicating standard error. A Student's t-test was calculated, and the asterisk indicates a p-value lower 0.05 . Experiments were independently reproduced at least three times with similar results

production of macroconidia. A tenfold increase of macroconidia production was noticed when $F$. graminearum was grown on VJ compared to mung-bean medium (Fig. 3). A test with Verticillium longisporum revealed that VJ medium was also suitable for production of $V$. longisporum conidia, with one agar plate yielding more than 600 Mio. spores on average.

\section{Comparison of ultrastructural features between CM- and VJ-grown S. indica}

To be able to identify possible differences in the ultrastructure of $S$. indica hyphae grown in CM and VJ medium, we prepared hyphae from $S$. indica liquid cultures grown for 11 days for electron microscopy. Hyphae from both media appeared very similar with respect to size and ultrastructural features. Samples from both media showed similar densities of nuclei, and of lipid bodies (Fig. 4).

\section{Interaction of VJS-grown S. indica chlamydospores with plants}

Using chlamydospores from VJS medium, we inoculated roots of 7-day-old barley plants. Staining of these roots 7 days after inoculation using WGA-Alexafluor 488, we observed fungal hyphae as well as chlamydospores colonizing the roots (Additional file 1: Fig. 3a), similar to published reports on barley root colonisation [5, 8]. We also used $S$. indica plugs from VJS medium and inoculated
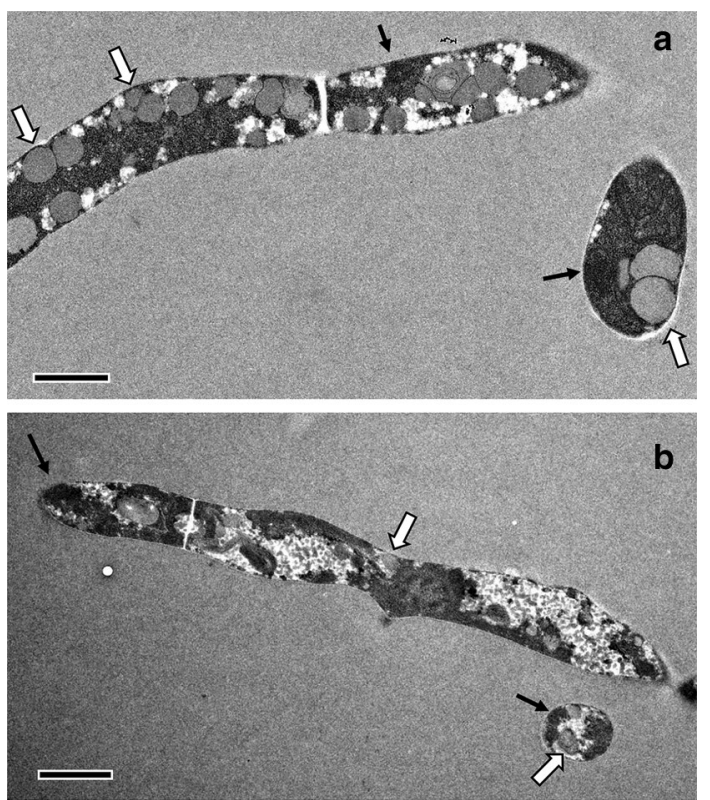

Fig. 4 Morphology of Serendipita indica hyphae from CM and $\mathrm{VJ}$ medium S. indica was grown in $100 \mathrm{ml}$ of CM medium a or VJ medium $\mathbf{b}$ for 10 days at room temperature $\left(20 \pm 3^{\circ} \mathrm{C}\right)$ on a shaker (70 rpm), and hyphae were subsequently harvested for fixation and preparation of electron microscopic images. Hyphal diameters were between 1 and $2 \mu \mathrm{M}$ in both media. Black arrows indicate nuclei, white arrows indicate lipid bodies. Bars represent $1 \mu \mathrm{m}$ a and $2 \mu \mathrm{m} \mathbf{b}$

7 day-old Arabidopsis thaliana (Col-0) seedlings growing on $1 / 2$ MS medium (Additional file 1: Fig. 3b). Presence of the fungus induced growth of the seedlings, visible 7 days after inoculation, comparable to published reports using S. indica fungal plugs grown on CM medium [22].

\section{Discussion}

We aimed at developing a medium that is easy to prepare, with only few and readily available, inexpensive ingredients which provides high inoculum yields for the fungus Serendipita indica, a fungus used as a model system to study plant-endophyte interactions in the lab and, due to its growth enhancing and plant strengthening properties, also studied in agricultural and biotechnological settings.

We optimised a medium for $S$. indica using commercially available vegetable juice as the main ingredient. Media based on the commercially available vegetable juice V8 have been shown to be useful for growth and sporangia induction of the plant pathogenic fungus Phytophthora [19], and are frequently used for conidia production in different ascomycetes including Fusarium culmorum, Parastagnospora nodorum and Pyrenophora tritici-repentis $[23,24]$.

In a series of experiments, we determined that a medium composition of $15 \%(\mathrm{v} / \mathrm{v}) \mathrm{V} 8$ vegetable juice and 
$0.1-0.4 \%(\mathrm{w} / \mathrm{v}) \mathrm{CaCO}_{3}$ are optimal, and superior to traditionally used complex medium (CM) with respect to the speed of hyphal growth and fungal biomass production in liquid culture. The observed high growth rate of $S$. indica did not depend on the brand of vegetable juice used, as comparable results were obtained when a different brand of vegetable juice was used (with tomato juice as the main component, and carrot, beetroot, celery, fermented cabbage, leek, parsley and lemon juice). Fungal inoculum obtained from liquid culture is frequently used for inoculation of larger plants, e.g. in pot experiments with barley or field experiments with wheat $[5,25]$ and we propose VJ medium for fast and efficient inoculum production. Pham et al. [15] reported yields between 3.67 and $2.39 \mathrm{~g}$ fresh weight per $100 \mathrm{ml}$ in CM medium, similar to our results shown in Fig. 1a. A report on enhancing S. indica biomass yield, using a modified Kaefer medium in a 14-L bioreactor, achieved production rates of $0.18 \mathrm{~g} \mathrm{~L}^{-1} \mathrm{~h}^{-1}$ [14]. This is about four times less than our results, using $300 \mathrm{ml}$ Erlenmeyer flasks with $100 \mathrm{ml}$ culture volume, yielding $21 \mathrm{~g}$ after 10 days of culture (Fig. 1).

In other experiments, e.g. when a defined amount of sterile fungal inoculum is required, $S$. indica chlamydospore preparations have been used [8, 26, 27]. For this purpose, chlamydospores can be easily retrieved by washing agar plates with water. A high chlamydospore yield was obtained with agar plates using VI medium supplemented with $4 \%$ Sucrose (VJS medium) (Fig. 2). Even higher chlamydospore yields may be obtained by using liquid culture in a bioreactor and subsequent removal of hyphae, as shown by [14], with yields up to $9.25^{*} 10^{7}$ spores per ml. Nevertheless, as fungal cultures on agar plates do not require specific equipment and hardly any hands-on time, it will probably remain the method of choice, at least for chlamydospore amounts required for research purposes.

We confirmed that $S$. indica inoculum produced with VJ medium has similar ultrastructural features as $\mathrm{CM}$ medium-grown fungus (Fig. 4). Roots were well colonised by the fungus after inoculation with VJS-grown fungus (Additional file 1: Fig. 3a), and S. indica-induced growth promotion was observed for Arabidopsis plants (Additional file 1: Fig. 3b), in line with previous reports on the properties of the fungal endophyte when propagated on CM medium $[22,28]$. We therefore suggest VIS medium for efficient chlamydospore production. In addition, VJ medium is also highly suitable for inoculum production of Fusarium graminearum and Verticillium longisporum: Macroconidia production of $F$. graminearum was about 10 times higher than with traditionally used mung bean agar (Fig. 3).

Some studies explained high growth rates on V8-based media with the presence of an inducing factor in V8 juice.
However, this inducing factor was later found to be rather a combination of factors required for induction of sexual development of C. neoformans [29]. We speculate that beneficial properties of VI medium for a high growth rate of $S$. indica are due to its complex nutrient composition and possibly the presence of secondary plant metabolites present in vegetable juice.

\section{Conclusions}

The optimized VJ medium described here was superior to complex media commonly used for production of fungal inoculum of the plant root endophytic model fungus Serendipita indica. Due to its ease of preparation and faster and more efficient inoculum production as compared with Kaefer-Hill medium, we expect this medium to become the medium of choice for $S$. indica. We further show that other fungal inocula used in plant pathology can also be produced efficiently using VJ medium. Besides its usefulness for research purposes due to the accessibility and relatively low price of its components, and the ease of preparation, it may also serve as a basis for the development of large-scale inoculum production for commercial purposes.

\section{Methods \\ Fungal materials}

Serendipita indica / Piriformospora indica isolate DSM11827 was obtained from the DSMZ (LeibnizInstitut DSMZ-Deutsche Sammlung von Mikroorganismen und Zellkulturen GmbH, Braunschweig, Germany). Fusarium strain F. graminearum was provided by Prof. Dr. R. Hückelhoven, Technical University Munich. Verticillium longisporum strain V143 [30] was obtained from Prof. W. Dröge-Laser, Würzburg University.

\section{Media preparation}

VJ medium was prepared using the commercially available vegetable juice V8 ('V8 Original Vegetable Juice'; Campbell Soup Company, Camden NJ, USA), which is mainly composed of tomato juice (86\%), supplemented with juice from beetroot, carrot, celery, spinach, parsley, lettuce and watercress, $\mathrm{NaCl}(6.8 \mathrm{~g} / \mathrm{L})$, and natural flavouring (www.v8juice.co.uk). For one liter of VJ medium, $850 \mathrm{ml}$ deionized, distilled water was supplemented with $150 \mathrm{ml}$ of $\mathrm{V} 8$ vegetable juice and $0.5 \mathrm{~g} \mathrm{CaCO}_{3}$. For VJ medium supplemented with sucrose (VJS medium), we used $20 \mathrm{mM}$ of $\mathrm{CaCO}_{3}$ and added $40 \mathrm{~g} / \mathrm{L}$ sucrose to the VJ medium.

For S. indica liquid culture, $300 \mathrm{ml}$ Erlenmeyer flasks containing $100 \mathrm{ml}$ of the VJ medium were used. The flasks were inoculated with 2 plugs $(5 \mathrm{~mm}$ diameter) from agar plates containing S. indica and kept at $20{ }^{\circ} \mathrm{C}$ on a rotary shaker $(70 \mathrm{rpm})$ for 10 days and harvested by 
filtration through miracloth. For production of S. indica chlamydospores on solid medium, VJS medium was supplemented with $1.2 \% \mathrm{w} / \mathrm{v}$ ) Phyto agar (Duchefa-biochemie.com). CM medium was based on the Aspergillus medium [31], as described in [15] without casamino acids and without agar for liquid medium, while 1.2\% Phyto agar was used for solid medium in Petri dishes. Mung bean agar was prepared as described in [32] with $1.2 \%$ Phyto agar. Arabidopsis seedlings were grown on $1 / 2$ MS medium (Murashige and Skoog medium basal mixture, including MES buffer, pH 5.8; Duchefa Biochemie B.V.) containing $0.5 \%$ sugar and $1 \%$ Phyto agar. To test the impact of myo-inositol, one liter of medium was prepared with distilled water, tryptone $(10 \mathrm{~g})$, yeast extract (5 g) and microelements (1 ml micro-element solution of the Aspergillus medium as described in [15]), supplemented with $10 \mathrm{~g}$ D-glucose or $10 \mathrm{~g}$ myo-inositol. All media (VJ medium, VJS medium, tryptone yeast agar, $\mathrm{CM}$ medium and MS medium) were autoclaved $\left(121^{\circ} \mathrm{C}\right.$, $20 \mathrm{~min}$ ) before use.

\section{Electron microscopy}

Spores and hyphae of $S$. indica grown in liquid medium for 11 days were centrifuged and the supernatant was removed. The resulting fungal material was fixated with $2.5 \%$ glutaraldehyde in cacodylate buffer overnight at $4{ }^{\circ} \mathrm{C}$. Further steps of electron microscopy sample preparation were as described in [33]. Images were recorded on a JEM-2100 transmission electron microscope (JEOL, Freising, Germany) at $200 \mathrm{kV}$ acceleration voltage with a F416 digital camera TVIPS TemCam F416 (Tietz Video and Images Processing Systems, Gauting, Germany).

\section{Fluorescence microscopy}

For microscopic investigation of fungal root colonization, barley roots were stained with Wheat Germ AgglutininAlexafluor 488 (WGA488; Molecular Probes; Invitrogen, www.invitrogen.com) as described in Deshmukh et al. (2006). Images were recorded on a confocal laser scanning microscope (Leica TCS SP5) using the bright field channel and a GFP filter set for detection of WGA488.

\section{Statistical analysis}

Data were subjected to one-way analysis of variance (ANOVA) followed by Tukey's post-hoc-test using IBM SPSS Statistics software (Ver. 25). For pairwise comparisons, Student's t-tests were calculated.

\section{Supplementary information}

Supplementary information accompanies this paper at https://doi. org/10.1186/s13007-020-00584-7.

Additional file 1: Figure 1. Diameter of $S$. indica hyphal growth on CM and $\mathrm{VJ}$ agar Diameters were determined 8 days after inoculation of $\mathrm{CM}$ or VJ plates with an $\mathrm{S}$. indica agar plug. Standard Petri dishes with a diameter of $9 \mathrm{~cm}$ were used and kept at room temperature $\left(20 \pm 3^{\circ} \mathrm{C}\right)$ either in the dark or in continuous light. Values are means from three plates, with error bars indicating standard deviation. The values were subjected to a one-way analysis of variance (ANOVA) followed by Tukey's post-hoc-test. Mean values significantly different at an alpha level of 0.05 are indicated by different letters. Experiments were independently reproduced two times with similar results. Figure 2. Effect of Myo-inositol and Glucose on S. indica growth a) Image of fungal growth on tryptone yeast agar supplemented with $10 \mathrm{~g} / \mathrm{L} \mathrm{D}$-glucose (left) or $10 \mathrm{~g} / \mathrm{L}$ myo-inositol (right) 8 days after inoculation with an S. indica agar plug. Standard Petri dishes with a diameter of $9 \mathrm{~cm}$ were used and kept at room temperature (20 $\pm 3^{\circ} \mathrm{C}$ ) in the dark. b) Fungal growth on agar plates supplied with 10 $\mathrm{g} / \mathrm{L}$ D-glucose or $10 \mathrm{~g} / \mathrm{L}$ myo-inositol 8 days after inoculation. Values are means from three plates, with error bars indicating standard deviation. A Student's t-test was calculated, and the asterisk indicates a p-value lower 0.05. Experiments were independently reproduced at least two times with similar results. Figure 3. Plant interaction of S. indica from VJ-grown inoculum a) Colonisation of barley roots with $\mathrm{S}$. indica after inoculation with spores prepared from VJS medium. Roots of $7 \mathrm{~d}$-old seedlings grown in hydroponic medium were inoculated and analyzed $7 \mathrm{~d}$ after inoculation. Germinated chlamydospores with their hyphae are visualized by staining root sections with a fluorescent dye-coupled wheat germ agglutinin (WGA-AF 488). Images were recorded using a confocal fluorescence microscope. Shown are the bright field image (right), fluorescence image (middle) and an overlay of these two images (left). The size bar represents $50 \mu \mathrm{m}$. b) Growth of Arabidopsis thaliana (Col-0) plants in the absence (left) or presence (right) of S. indica. One-week-old Arabidopsis seedlings growing in Petri dishes ( $9 \mathrm{~cm}$ diameter) on $1 / 2$ MS medium were inoculated with a S. indica mycelial plug obtained from VJS medium. Plates were photographed after 3 weeks of growth under short-day conditions ( $8 \mathrm{~h}$ light / $16 \mathrm{~h}$ dark, $\left.22^{\circ} \mathrm{C}\right)$.

\section{Acknowledgements}

We thank Paula Puente for preparing S. indica-colonised barley plants. Fusarium graminearum was kindly provided by Prof. Dr. R. Hückelhoven, Technical University Munich. Verticillium longisporum was kindly provided by Prof. W. Dröge-Laser, Würzburg University.

\section{Authors' contributions}

$\mathrm{MO}$ and FW designed the study. MO, CS and FW performed experiments, all authors analyzed and interpreted the data. The manuscript was prepared by $\mathrm{MO}$ and FW. All authors read and approved the final manuscript.

\section{Funding}

This publication was funded by the German Research Foundation (DFG) and the University of Wuerzburg in the funding program Open Access Publishing. The funding bodies had no role in the design of the study and in the collection, analysis, and interpretation of data and in writing the manuscript.

\section{Availability of data and materials}

All data generated or analysed during this study are included in this published article and its supplementary information files.

Ethics approval and consent to participate

Not applicable.

\section{Consent for publication}

Not applicable.

Competing interests

The authors declare that they have no competing interests. 


\section{Author details}

1 Julius-Von-Sachs Institute of Biosciences, Biocenter, Pharmaceutical Biology, Julius-Maximilians-Universität Würzburg, Julius-von-Sachs-Platz 2, 97082 Würzburg, Germany. ${ }^{2}$ Imaging Core Facility, Theodor-Boveri Institute of Biosciences, Biocenter, Julius-Maximilians-Universität Würzburg, Am Hubland, 97074 Würzburg, Germany.

Received: 17 October 2019 Accepted: 11 March 2020

Published online: 16 March 2020

\section{References}

1. Verma S, Varma A, Rexer KH, Hassel A, Kost G, Sarbhoy A, Bisen P, Butehorn B, Franken P. Piriformospora indica, gen. et sp. nov, a new root-colonizing fungus. Mycologia. 1998;90(5):896-903.

2. Oberwinkler F, Riess K, Bauer R, Selosse M-A, Weiß M, Garnica S, Zuccaro A. Enigmatic Sebacinales. Mycol Prog. 2013;12(1):1-27.

3. Weiss M, Waller F, Zuccaro A, Selosse MA. Sebacinales - one thousand and one interactions with land plants. New Phytol. 2016;211(1):20-40.

4. Varma A, Savita V, Sudha J, Sahay N, Butehorn B, Franken P. Piriformospora indica, a cultivable plant-growth-promoting root endophyte. Appl Environ Microbiol. 1999;65(6):2741-4.

5. Waller F, Achatz B, Baltruschat H, Fodor J, Becker K, Fischer M, Heier T, Huckelhoven R, Neumann C, von Wettstein D, et al. The endophytic fungus Piriformospora indica reprograms barley to salt-stress tolerance, disease resistance, and higher yield. Proc Natl Acad Sci USA. 2005;102(38):13386-91.

6. Franken P. The plant strengthening root endophyte Piriformospora indica: potential application and the biology behind. Appl Microbiol Biotechnol. 2012;96(6):1455-64.

7. Jogawat A, Vadassery J, Verma N, Oelmuller R, Dua M, Nevo E, Johri AK. PiHOG 1, a stress regulator MAP kinase from the root endophyte fungus Piriformospora indica, confers salinity stress tolerance in rice plants. Sci Rep. 2016;6:36765.

8. Deshmukh S, Huckelhoven R, Schafer P, Imani J, Sharma M, Weiss M, Waller F, Kogel KH. The root endophytic fungus Piriformospora indica requires host cell death for proliferation during mutualistic symbiosis with barley. Proc Natl Acad Sci USA. 2006;103(49):18450-7.

9. Zuccaro A, Lahrmann U, Guldener U, Langen G, Pfiffi S, Biedenkopf D, Wong P, Samans B, Grimm C, Basiewicz M, et al. Endophytic life strategies decoded by genome and transcriptome analyses of the mutualistic root symbiont Piriformospora indica. PLoS Pathog. 2011;7(10):e1002290.

10. Stein E, Molitor A, Kogel KH, Waller F. Systemic resistance in Arabidopsis conferred by the mycorrhizal fungus Piriformospora indica requires jasmonic acid signaling and the cytoplasmic function of NPR1. Plant Cell Physiol. 2008;49(11):1747-51.

11. Qiang X, Weiss M, Kogel KH, Schafer P. Piriformospora indica-a mutualistic basidiomycete with an exceptionally large plant host range. Mol Plant Pathol. 2012;13(5):508-18.

12. Baldi A, Jain A, Gupta N, Srivastava AK, Bisaria VS. Co-culture of arbuscular mycorrhiza-like fungi (Piriformospora indica and Sebacina vermifera) with plant cells of Linum album for enhanced production of podophyllotoxins: a first report. Biotechnol Lett. 2008;30(9):1671-7.

13. Baishya D, Deka P, Kalita MC. In vitro co-cultivation of Piriformospora indica filtrate for improve biomass productivity in Artemisia annua(L.). Symbiosis. 2015;66(1):37-46.

14. Kumar V, Sahai V, Bisaria VS. High-density spore production of Piriformospora indica, a plant growth-promoting endophyte, by optimization of nutritional and cultural parameters. Bioresour Technol. 2011;102(3):3169-75.

15. Pham GH, Kumari R, Singh A, Malla R, Prasad R, Sachdev M, Kaldorf M, Buscot F, Oelmüller R, Hampp R, Saxena AK, Rexer K-H, Kost G, Varma A. Axenic culture of symbiotic fungus Piriformospora indica. In: Plant surface microbiology. 2004; p. 593-612.
16. Kumar V, Sahai V, Bisaria VS. Production of amylase and chlamydospores by Piriformospora indica, a root endophytic fungus. Biocatal Agric Biotechnol. 2012;1(2):124-8.

17. Hill TW, Kafer E. Improved protocols for Aspergillus minimal medium: trace element and minimal medium salt stock solutions. Fungal Genet Newsletter. 2001;48(1):20-1.

18. Arora M, Divya K, Kharkwal A, Varma A. Use of locally available jaggery for development of a cost effective media for mass cultivation of Piriformospora indica-a step forward towards commercialization. Int J Curr Res. 2014;6(11):9531-5.

19. Ayers WA. Induction of sporangia in Phytophthora cinnamomi by a substance from bacteria and soil. Can J Microbiol. 1971;17(12):1517-23.

20. Clements RS Jr, Darnell B. Myo-inositol content of common foods: development of a high-myo-inositol diet. Am J Clin Nutr. 1980;33(9):1954-67.

21. Xue C, Liu T, Chen L, Li W, Liu I, Kronstad JW, Seyfang A, Heitman J. Role of an expanded inositol transporter repertoire in Cryptococcus neoformans sexual reproduction and virulence. MBio. 2010;1(1):e00084-e10.

22. Peskan-Berghofer T, Shahollari B, Giong PH, Hehl S, Markert C, Blanke V, Kost G, Varma A, Oelmuller R. Association of Piriformospora indica with Arabidopsis thaliana roots represents a novel system to study beneficial plant-microbe interactions and involves early plant protein modifications in the endoplasmic reticulum and at the plasma membrane. Physiol Plant. 2004;122(4):465-77.

23. Osman M, He X, Benedettelli S, Ali S, Singh PK. Identification of new sources of resistance to fungal leaf and head blight diseases of wheat. Eur J Plant Pathol. 2016;145(2):305-20.

24. Beccari G, Prodi A, Pisi A, Nipoti P, Onofri A, Nicholson P, Pfohl K, Karlovsky P, Gardiner DM, Covarelli L. Development of three fusarium crown rot causal agents and systemic translocation of deoxynivalenol following stem base infection of soft wheat. Plant Pathol. 2018;67(5):1055-65.

25. Serfling A, Wirsel SG, Lind V, Deising HB. Performance of the biocontrol fungus Piriformospora indica on wheat under greenhouse and field conditions. Phytopathology. 2007;97(4):523-31.

26. Pedrotti L, Mueller MJ, Waller F. Piriformospora indica root colonization triggers local and systemic root responses and inhibits secondary colonization of distal roots. PLoS ONE. 2013;8(7):e69352.

27. Qiang X, Zechmann B, Reitz MU, Kogel KH, Schafer P. The mutualistic fungus Piriformospora indica colonizes Arabidopsis roots by inducing an endoplasmic reticulum stress-triggered caspase-dependent cell death. Plant Cell. 2012;24(2):794-809.

28. Schäfer P, Pfiffi S, Voll LM, Zajic D, Chandler PM, Waller F, Scholz U, Pons-Kühnemann J, Sonnewald S, Sonnewald U, et al. Manipulation of plant innate immunity and gibberellin as factor of compatibility in the mutualistic association of barley roots with Piriformospora indica. Plant J. 2009;59(3):461-74.

29. Kent CR, Ortiz-Bermúdez P, Giles SS, Hull CM. Formulation of a defined V8 medium for induction of sexual development of Cryptococcus neoformans. Appl Environ Microbiol. 2008;74(20):6248-53.

30. Zeise K, von Tiedemann A. Host specialization among vegetative compatibility groups of Verticillium dahliae in relation to Verticillium longisporum. J Phytopathol. 2002;150(3):112-9.

31. Käfer E. Meiotic and mitotic recombination in aspergillus and its chromosomal aberrations. In: Caspari EW, editor. Advances in Genetics, vol. 19. Cambrige: Academic Press; 1977. p. 33-131.

32. Buerstmayr $H$, Stierschneider M, Steiner B, Lemmens M, Griesser M, Nevo E, Fahima T. Variation for resistance to head blight caused by Fusarium graminearum in wild emmer (Triticum dicoccoides) originating from Israel. Euphytica. 2003;130(1):17-23.

33. Prüfert K, Vogel A, Krohne G. The lamin CxxM motif promotes nuclear membrane growth. J Cell Sci. 2004;117(25):6105-16.

\section{Publisher's Note}

Springer Nature remains neutral with regard to jurisdictional claims in published maps and institutional affiliations. 